\title{
VALEUR FOURRAGERE DES ESPECES PRELEVEES PAR GAZELLA CUVIERI OGILBY, 1841 AU NIVEAU DU DJEBEL METLILI (ALGERIE)
}

\author{
FEED VALUE OF SPECIES LEVIED BY GAZELLA CUVIERI OGILBY, 1841 AT THE \\ DJEBEL METLILI (ALGERIA)
}

Arbouche, Y. ${ }^{1}$, Arbouche, H.S. ${ }^{2 *}$, Arbouche, F. ${ }^{2 A}$ et Arbouche, R. ${ }^{2 B}$

\begin{abstract}
${ }^{1}$ Agronomie Université Ferhat Abbas. Sétif-1900. Algérie. yas.arbouche@yahoo.fr
${ }^{2}$ Agronomie Centre Universitaire. El Tarf. Algérie. *arbouchehs@yahoo.fr; Aarbouchefodil@yahoo.fr; Brafik_arbouche@yahoo.fr
\end{abstract}

\section{MOTS CLÉS ADDITIONNELLES}

Régime alimentaire.

\section{RÉSUMÉ}

Les espèces végétales intéressant le régime alimentaire de la Gazelle de Cuvier ont été déterminées par observation directe au niveau de son aire de répartition. Pour la strate herbacée, les graminées sont majoritaires (cinq espèces) et les légumineuses peu représentées (une espèce: Medicago sativa). Cette strate contribue à un niveau énergétique appréciable aux stades débourrement et floraison, mais peu conséquent au stade fruit. A ce stade, les chaméphytes ont aussi des valeurs fourragères faibles $(0,00$ à 0,33 UF/kg de MS).

\section{SUMMARY}

The plants species interesting the diet of the gazelle of Cuvier were determined by direct observation at the level of its area of distribution. For herbaceous plants, grasses are a majority (five species) and leguminous little represented (one species: Medicago sativa). This stratum contribue to an energy level interesting in the stages of preflowering and flowering, but has little importance at the stage of fruit. At this stage, the chamephytes have also weak fodder values $(0,00$ to $0,33 \mathrm{UF} / \mathrm{kg}$ of DM).

\section{INTRODUCTION}

La Gazelle de Cuvier est endémique à l'Afrique du Nord (Abáigar et Cano, 2005). C'est une espèce protégée et menacée d'extinction en Algérie. Animal rustique, il

Recibido: 14-12-10. Aceptado: 20-12-10.

\section{AdDitional KeYWORDS \\ Feeding at grazing.}

s'adapte et supporte les conditions climatiques rigoureuses telles que les hautes chaleurs et le grand froid (Talbi,1989). Plusieurs études ont mentionné les différentes espèces végétales broutées entrant dans le régime alimentaire de la Gazelle de Cuvier (Gazella cuvieri, Ogylbi 1841). En Espagne, Escos Quilez (1992); en Tunisie, Karem et al., 1993; en Algérie, Talbi (1989) et au Maroc, Cuzin (1996). La zone d'étude comprise dans ce territoire prospecté, fait état d'un petit groupe de 15 individus.

\section{MATÉRIELETMÉTHODES}

\section{ZONED'ÉTUDE}

Le parc national du Belezma (P.N.B) se situe sur la partie orientale de l'Algérie du Nord. L'étude porte sur la région du Djebel Metlili qui couvre la partie sud du massif des Aurès. Elle est localisée dans l'étage bioclimatique semi-aride, est caractérisée par un hiver frais et pluvieux et un été chaud et sec. La température moyenne annuelle est de $14^{\circ} \mathrm{C}$ avec un maximum de $25,5^{\circ} \mathrm{C}$ et un minimum de $5,1^{\circ} \mathrm{C}$. Les précipitations moyennes annuelles sont de $300 \mathrm{~mm}$.

\section{RÉGIMEALIMENTAIRE}

Il a été étudié par l'observation directe de l'abroutissement des végétaux par la 
Gazelle de Cuvier Grâce à une végétation arborée peu dense, l'observation des différentes espèces végétales prélevées par la Gazelle de Cuvier a été réalisée par la mise en place de sites d'observations sur une période d'une année à raison de deux semaines par mois (début et fin de mois), à l'aide de télescopes. Pour la pelouse, ont été pris en considération les espèces dominantes qui la composent.

\section{PrélèVements et ANALYSES}

Trois prélèvements ont été réalisés suivant le stade phénologique de chaque espèce. Pour le choix des espèces entrant dans le régime alimentaire, deux facteurs sont pris en considération : la fréquence du broutement de l'espèce végétale et l'importance de la densité pour les arbustes et le taux de recouvrement pour les herbacées et les chaméphytes. Un stade phénologique est considéré comme effectif, lorsque $80 \%$ de l'ensemble des végétaux composant l'espèce a atteint le dit stade (Demarquilly, 1981).

Les analyses chimiques ont été effectuées selon la méthode édictée par AOAC (1975). Concernant la digestibilité, la méthode utilisée est celle décrite par Aufrère (1982). Les valeurs fourragères ont été calculées suivant les équations de Sauvant et al. (2004). Le test statistique a été effectué à l'aide du logiciel Statistica 6.0 ( StatSoft Inc., 2001).

\section{RÉSULTATSETDISCUSSION}

\section{ESPĖCESPRÉLEVÉES}

La préhension par la Gazelle de Cuvier d'espèces végétales est une fonction du milieu comme le signalent plusieurs auteurs notamment, Talbi (1989) qui par observation directe au niveau du Djebel El-Achch (Wilaya de Saida) a mentionné Olea europea, Arbutus unedo, Pistacia lentiscus, Phillyrea angustifolia, Quercus coccifera, Calycotome spinosa, Lonicera implexa, Lavandula stoechas et Lycium arabicum.

\section{COMPOSITION CHIMIQUE DES ESPĖCES VÉGÉTALES CONSOMMÉES}

La strate herbacée: Du stade débourrement au stade fruit, la matière sèche évolue de façon significative, elle passe en moyenne de 25,3 à $94,5 \%$ pour toute espèce confondue (tableau I). Les graminées au stade fruit contribuent largement à l'augmentation quantitative de la matière sèche par rapport à la légumineuse (Medicago sativa) qui n'apporte que $39,2 \%$.

Les chaméphytes: Pour toutes les espèces, le taux de matière sèche du stade végétatif au stade grain mature est important (au-delà de 50\%) (tableau I). La matière azotée totale varie entre 0,9 et $20,5 \%$ de MS, avec un apport maximal au stade floraison (10,5 à 17,0\% de MS).

La strate arbustive: Les taux de matière sèche et de matière organique sont maximaux au stade fruit pour l'ensemble des espèces composant la strate arbustive (tableau I). Le taux de matière azotée est maximal au stade floraison et varie entre 10,9 à 14,3\% de MS. Le maximum étant amené par Phillyrea media.

\section{DIGESTIBILITÉ}

Les résultats de la digestibilité (CUD) de la matière organique concernant les strates arbustives et chaméphytes, sont conformes avec ceux de Le Houerou (1980), qui signale une grande variabilité du coefficient d'utilisation digestive (CUD) de la matière organique de l'ordre de 30 à $72 \%$ pour les espèces ligneuses. Pour la strate herbacée, les résultats des différentes espèces d'herbacées étudiées par Andrieu et al. (1988), présentent des CUD plus élevés (70 à $80 \%)$.

\section{LES VALEURS FOURRAGĖRES DES DIFFÉ- RENTESESPĖCESBROUTÉES}

Les teneurs en UF les plus importantes sont celles de Medicago sativa avec 0,66/ $\mathrm{kg}$ de MS au stade débourrement et $0,40 / \mathrm{kg}$ de MS au stade fruit. Pour la strate des chaméphytes, au stade végétatif, Artemisia 


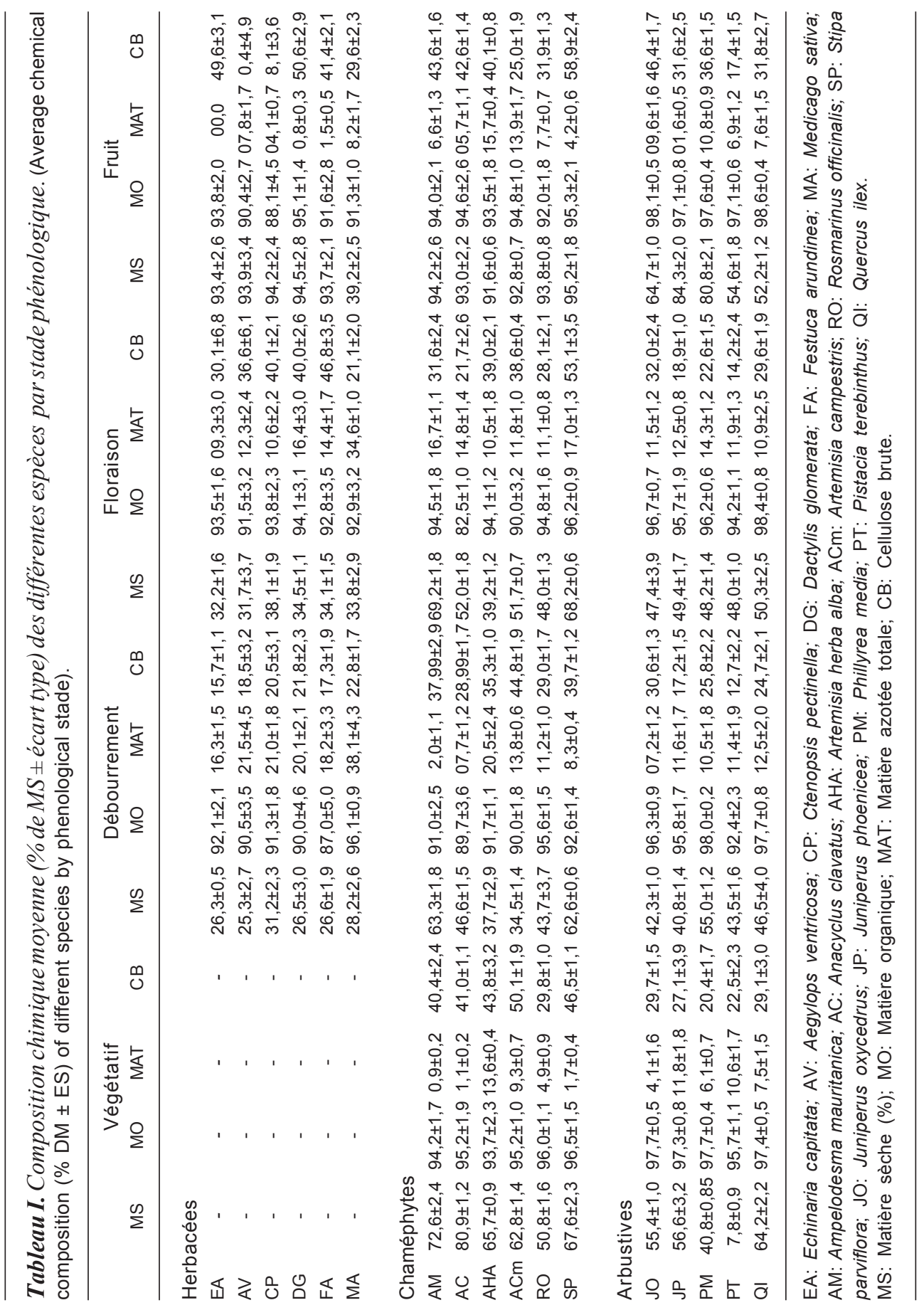




\section{ARBOUCHE, ARBOUCHE, ARBOUCHE ET ARBOUCHE}

herba alba contribue largement à la valorisation des UF avec $0,57 / \mathrm{kg}$ de MS. Au stade débourrement, Rosmarinus officinalis et Stipa parviflora avec respectivement 0,57 et $0,58 / \mathrm{kg}$ de $\mathrm{MS}$, sont les espèces qui contribuent le plus au développement de la gazelle de Cuvier. Au stade floraison, Rosmarinus officinalis avec $0,65 \mathrm{UF} / \mathrm{kg}$ de MS est la plus valorisante pour ce stade. Pour les arbustives, les valeurs fourragères sont optimums au stade débourrement avec un maximum de 0,75/kg de MS pour Phillyrea media.

\section{BIBLIOGRAPHIE}

AOAC. 1975. Official methods of analysis. $12^{\text {th }}$ edition. Washington, DC

Abaigar, T. y Cano, M. 2005. Conservación y manejo de la Gacela de Cuvier (Gazella cuvieri Ogilby, 1841) en cautividad. Registro internacional. Instituto de Estudios Almerienses. Almería.

Andrieu, J., Demarquilly, C. et Sauvant, D. 1988. Tables de la valeur nutritive des aliments. In: Jarrige (Ed.). Alimentation des bovins, ovins et caprins. INRA. Paris.

Aufrere, J. 1982. Etude de la prévision de la digestibilité des fourrages par une méthode enzymatique (pepsine-cellulase). Ann Zoot, 31: 111-130.

Cuzin, F. 1996. Répartition actuelle et statut des grands mammifères sauvages du Maroc (Primates, Carnivores, Artiodactyles). Mammalia, 60: 101-124.

Demarquilly, C. 1981. Valeur alimentaire de l'herbe dans les conditions du pâturage. Fourrages, 85: $59-72$.

Escos Quilez, J. 1992. Gazella cuvieri studbook.

\section{CONCLUSION}

Le régime alimentaire de la gazelle de Cuvier au niveau du parc national du Belezma (Djebel Metlili) concerne trois strates: herbacée, chaméphyte et arbustives. Les valeurs énergétiques des espèces broutées sont complémentaires en fonction des stades phénologiques. Les stades fruit des espèces herbacées et chaméphytes n'assurent que très peu d'apports en UF, lesquels sont compensés par les arbustives.

Instituto de Estudios Almerienses. Almería.

Karem, A., Ksantini, M., Schoenenberger, A. et Waibel, T. 1993. Contribution à la régénération de la végétation dans les parcs nationaux en Tunisie aride. GTZ. Eschborn.

Le Houerou, H.N. 1980. Les fourrages ligneux en Afrique. Centre international pour l'élevage en Afrique. Addis-Abeba.

Sauvant, D., Perez, J.M. et Tran, G. 2004. Tables de composition et de valeur nutritive des matières premières destinées aux animaux d'élevage. Porcs, volailles, bovins, ovins, caprins, lapins, chevaux et poisons $2^{e}$ éd. Revue et corrigé INRA. Paris. France.

StatSoft Inc. 2001. Statistica. Data analysis software system. Versión 6. USA. www.statsoft.com $(10 / 03 / 10)$

Talbi, K. 1989. Etude éco-éthologique de la Gazelle du Cuvier (Gazella cuvieri, Ogilby 1841) dans la zone de Djebel El Achch. Wilaya de Saïda. Thèse de Magistère. Institut National Agronomique. Alger.

Archivos de zootecnia vol. 61, núm. 233, p. 148. 